\title{
Fusion Graphs, Region Merging and Watersheds
}

\author{
Jean Cousty, Gilles Bertrand, Michel Couprie, and Laurent Najman \\ Institut Gaspard-Monge \\ Laboratoire A2SI, Groupe ESIEE \\ Cité Descartes, BP99 93162 Noisy-le-Grand Cedex France \\ \{j.cousty, g.bertrand, m.couprie, 1.najman\}@esiee.fr
}

\begin{abstract}
Region merging methods consist of improving an initial segmentation by merging some pairs of neighboring regions. We consider a segmentation as a set of connected regions, separated by a frontier. If the frontier set cannot be reduced without merging some regions then we call it a watershed. In a general graph framework, merging two regions is not straightforward. We define four classes of graphs for which we prove that some of the difficulties for defining merging procedures are avoided. Our main result is that one of these classes is the class of graphs in which any watershed is thin. None of the usual adjacency relations on $\mathbb{Z}^{2}$ and $\mathbb{Z}^{3}$ allows a satisfying definition of merging. We introduce the perfect fusion grid on $\mathbb{Z}^{n}$, a regular graph in which merging two neighboring regions can always be performed by removing from the frontier set all the points adjacent to both regions.
\end{abstract}

\section{Introduction}

Region merging methods [1,2 consist of improving an initial segmentation by progressively merging pairs of neighboring regions until a certain criterion is satisfied. From a grayscale image, the watershed transform [3, 4, 5, 6, 7, 8,, , produces a set of connected regions separated by a divide. Therefore it has long been used as an entry point for region merging methods [10. In a general graph framework, a watershed may be thought of as a "separating set" of vertices which cannot be reduced without merging some connected components of its complementary set.

A first question arises when dealing with watersheds on a graph. Given a subset of $\mathbb{Z}^{2}$ equipped with the 4-adjacency relation, we observe that a watershed may contain some "interior points", i.e., points which are not adjacent to any point outside the watershed (see for example the points $w$ and $z$ on Fig. 11.). On the other hand, such interior points do not seem to appear in any watershed on 8-connected graphs. Are the watersheds on these graphs always thin? We will prove that it is indeed true. More interestingly, we provide in this paper a framework to study the property of thinness of watersheds in any kind of graph, and we identify the class of graphs in which any watershed is necessarily thin. 


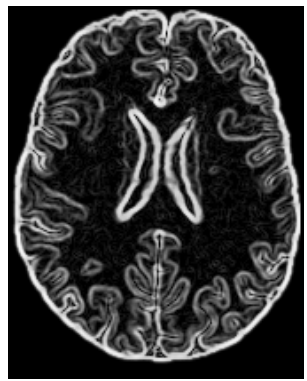

(a)

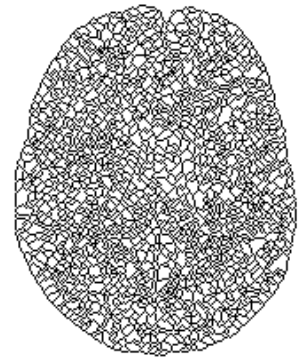

(b)

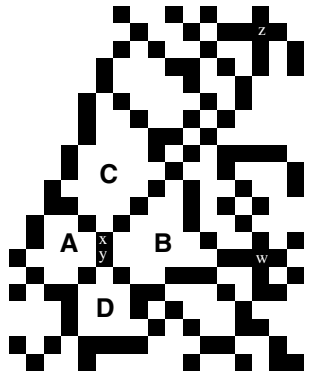

(c)

Fig. 1. (a): Original image (cross-section of a brain, after applying a gradient operator). $(b)$ : Watershed of $(a)$ with the 4-adjacency (in black). (c): A zoom on a part of $(b)$. The points $z$ and $w$ are interior points.

Let us now turn back to the region merging problem. What happens if we want to merge a couple of neighboring regions $A$ and $B$, and if each pixel adjacent to these two regions is also adjacent to a third one, which is not wanted in the merging? Fig. 1 illustrates such a situation, where $x$ is adjacent to regions $A, B, C$ and $y$ to $A, B, D$. This problem has been identified in particular by T. Pavlidis (see 2, section 5.6: "When three regions meet"), and has been dealt with in some practical ways, but until now a systematic study of properties related to merging in graphs has not been done. A major contribution of this article is the definition and the study of four classes of graphs, with respect to the possibility of "getting stuck" in a merging process (Sec. 2, Sec. 3). In particular, we say that a graph is a fusion graph if any region $A$ in this graph can always be merged with another region $B$, without problems with other regions. The most striking outcome of this study is that the class of fusion graphs is precisely the class of graphs in which any watershed is thin (Th. 3). We also provide some local characterizations for two of these four classes of graphs, and prove that the two other ones cannot be locally characterized (Sec. (4).

In one of the classes of graphs introduced in Sec. 3, that we call the class of perfect fusion graphs, any pair of neighboring regions can always be merged, without problems with other regions, by removing all pixels adjacent to both regions. Using our framework, we analyze the status of the graphs which are the most widely used for image analysis, namely the graphs corresponding to the 4and the 8-adjacency in $\mathbb{Z}^{2}$ and to the 6 - and the 26 -adjacency in $\mathbb{Z}^{3}$ (Sec. 5). We show that none of these classical graphs is a perfect fusion graph. Last, but not least, in Sec. 66 we introduce a graph on $\mathbb{Z}^{n}$ (for any $n$ ) that we call the perfect fusion grid, which is indeed a perfect fusion graph, and which is "between" the direct adjacency graph (which generalizes the 4-adjacency to $\mathbb{Z}^{n}$ ) and the indirect adjacency graph (which generalizes the 8-adjacency). Furthermore, in a forthcoming paper, we intend to prove that this $n$-dimensional grid is the unique grid (up to a translation) that possesses those two properties.

The proofs of the properties presented in this paper are given in an extended version [11. 


\section{Graphs and Watershed}

Let $E$ be a set, we write $X \subseteq E$ if $X$ is a subset of $E$, we write $X \subset E$ if $X$ is a proper subset of $E$, i.e., if $X$ is a subset of $E$ and $X \neq E$. We denote by $\bar{X}$ the complementary set of $X$ in $E$, i.e., $\bar{X}=E \backslash X$. If $E$ is a finite set, we denote by $|E|$ the number of elements of $E$ and by $2^{E}$ the set composed of all the subsets of $E$.

We define a graph as a pair $(E, \Gamma)$ where $E$ is a finite set and $\Gamma$ is a binary relation on $E$ (i.e., $\Gamma \subseteq E \times E$ ), which is reflexive (for all $x$ in $E,(x, x) \in \Gamma$ ) and symmetric (for all $x, y$ in $E,(y, x) \in \Gamma$ whenever $(x, y) \in \Gamma$ ). Each element of $E$ is called a vertex or a point. We will also denote by $\Gamma$ the map from $E$ to $2^{E}$ such that, for all $x \in E, \Gamma(x)=\{y \in E \mid(x, y) \in \Gamma\}$. If $y \in \Gamma(x)$, we say that $y$ is adjacent to $x$. We define also the map $\Gamma^{*}$ such that for all $x \in E, \Gamma^{*}(x)=\Gamma(x) \backslash\{x\}$. Let $X \subseteq E$, we define $\Gamma(X)=\cup_{x \in X} \Gamma(x)$, and $\Gamma^{*}(X)=\Gamma(X) \backslash X$. If $y \in \Gamma(X)$, we say that $y$ is adjacent to $X$. If $X, Y \subseteq E$ and $\Gamma(X) \cap Y \neq \emptyset$, we say that $Y$ is adjacent to $X$.

Let $G=(E, \Gamma)$ be a graph and let $X \subseteq E$, we define the subgraph of $G$ induced by $X$ as the graph $G_{X}=(X, \Gamma \cap[X \times X])$. In this case, we also say that $G_{X}$ is a subgraph of $G$. Let $G^{\prime}=\left(E^{\prime}, \Gamma^{\prime}\right)$ be a graph, we say that $G$ and $G^{\prime}$ are isomorphic if there exists a bijection $f$ from $E$ to $E^{\prime}$ such that, for all $x, y \in E$, $y$ belongs to $\Gamma(x)$ if and only if $f(y)$ belongs to $\Gamma^{\prime}(f(x))$.

Let $(E, \Gamma)$ be a graph, let $X \subseteq E$, a path in $X$ is a sequence $\pi=\left\langle x_{0}, \ldots, x_{l}\right\rangle$ such that $x_{i} \in X, i \in[0, l]$, and $x_{i} \in \Gamma\left(x_{i-1}\right), i \in[1, \ldots, l]$. We also say that $\pi$ is a path from $x_{0}$ to $x_{l}$ in $X$ and that $x_{0}$ and $y_{l}$ are linked for $X$. We say that $X$ is connected if any $x$ and $y$ in $X$ are linked for $X$.

Let $Y \subseteq X$. We say that $Y$ is a connected component of $X$, or simply a component of $X$, if $Y$ is connected and if $Y$ is maximal for this property, i.e., if $Z=Y$ whenever $Y \subseteq Z \subseteq X$ and $Z$ connected.

We denote by $\mathcal{C}(X)$ the set of all the connected components of $X$.

In this paper, we study some thinness properties of watersheds in graphs.

Definition 1. Let $(E, \Gamma)$ be a graph. Let $X \subseteq E$, the interior of $X$ is the set $\operatorname{int}(X)=\{x \in X \mid \Gamma(x) \subseteq X\}$. We say that the set $X$ is thin if $\operatorname{int}(X)=\emptyset$.

Let us recall the definition of line graphs ( [12]). This class of graphs allows to link the framework developed in this paper and the approaches of watershed and region merging based on edges rather than vertices, i.e. when regions are separated by a set of edges.

Definition 2. Let $(E, \Gamma)$ be a graph. The line graph of $(E, \Gamma)$ is the graph $\left(E^{\prime}, \Gamma^{\prime}\right)$ such that $E^{\prime}=\Gamma^{\star}$ and $(u, v)$ belongs to $\Gamma^{\prime}$ whenever $u \in \Gamma^{\star}, v \in \Gamma^{\star}$, and $u, v$ share a vertex of $E$.

We say that a graph $\left(E^{\prime}, \Gamma^{\prime}\right)$ is a line graph if there exists a graph $(E, \Gamma)$ such that $\left(E^{\prime}, \Gamma^{\prime}\right)$ is isomorphic to the line graph of $(E, \Gamma)$.

In Fig. 2, we show a graph and its line graph. All graphs are not line graphs, in other words, there exist some graphs which are not the line graphs of any graph. The following theorem allows to characterize line graphs. 


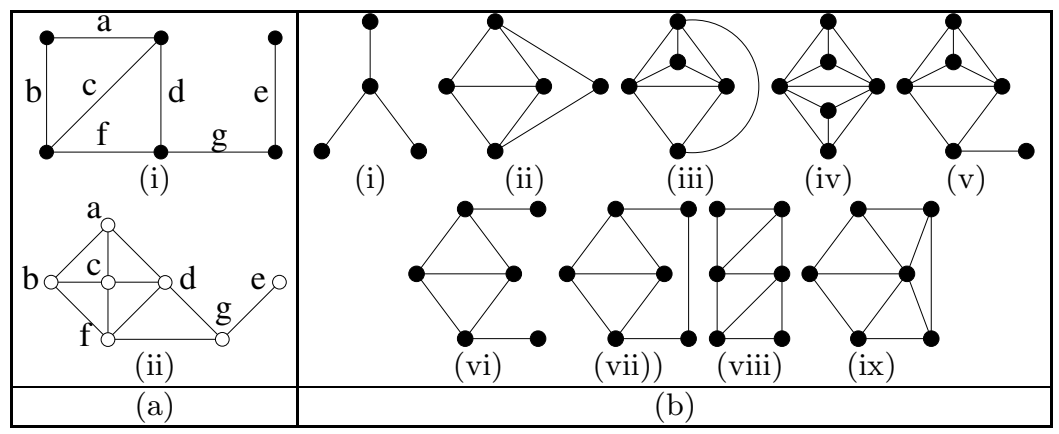

Fig. 2. (a): A graph (i) and its line graph (ii); (b): graphs for characterization of line graphs

Theorem 1 ( [12]). A graph $G$ is a line graph if and only if none of the graphs of Fig. [2b is a subgraph of $G$.

Important Remark. From now, when speaking about a graph $(E, \Gamma)$, we will assume for simplicity that $E$ is non-empty and connected.

Notice that, nevertheless, the subsequent definitions and properties may be easily extended to non-connected graphs.

We now introduce notions for watersheds in graphs.

Definition 3. Let $(E, \Gamma)$ be a graph. Let $X \subseteq E$, and let $p \in X$. We say that:

- $p$ is an inner point (for $X$ ) if $p \in \operatorname{int}(X)$.

- $p$ is W-simple (for $X$ ) if $p$ is adjacent to exactly one component of $\bar{X}$.

In this definition and the following ones, the prefix "W-" stands for watershed. In Fig. 3a, $x$ is a W-simple point for the set $X$ constituted by the black vertices, and $y$ is an inner point.

Definition 4. Let $G=(E, \Gamma)$ be a graph. A set $X \subseteq E$ is a watershed (in $G$ ) if there is no point $W$-simple for $X$.

$A$ watershed $X$ is non-trivial if $X \neq \emptyset$ and $X \neq E$.

Figs. 3b,c,d,e illustrate the notion of watershed. It can be seen that a watershed $X$ is non-trivial if and only if $|\mathcal{C}(\bar{X})| \geq 2$. A watershed is a set which contains no $\mathrm{W}$-simple point, but some of the examples given below show that such a set is not always thin (in the sense of Def. 11). Fig. 3b is an example of watersheds which is thin: the set of black points has no W-simple point and no inner point. The sets of black and gray points in Figs. 3r, d,e are three examples of non-thin watersheds.

\section{Merging}

Consider the graph $(E, \Gamma)$ depicted in Fig. 4a, where a subset $X$ of $E$ (black vertices) separates its complementary set $\bar{X}$ into four connected components. If 


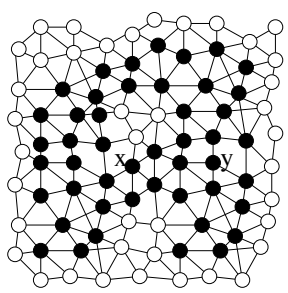

(a)

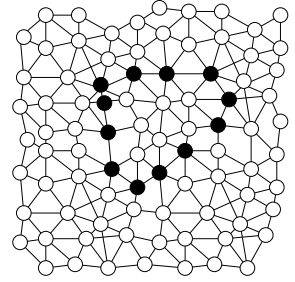

(b)

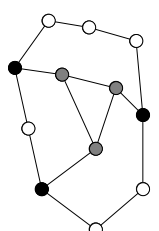

(c)

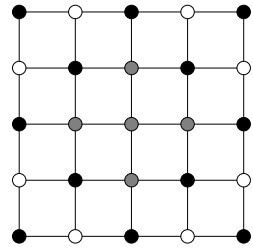

$(d)$

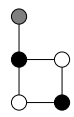

(e)

Fig. 3. Illustration of watershed. (a) A graph $(E, \Gamma)$ and a subset $X$ of $E$ (in black). (b) A thin watershed (black points). $(c-e)$ : The subset $X$ represented by black and gray points is a watershed which is not thin: $\operatorname{int}(X)$ is depicted by the gray points.

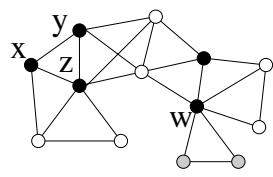

(a)

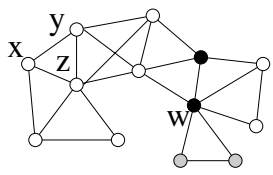

(b)

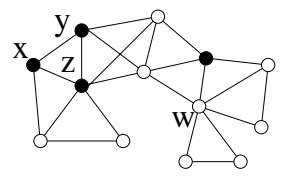

(c)

Fig. 4. Illustration of merging. (a): A graph $(E, \Gamma)$ and a subset $X$ of $E$ (black points). (b): The black points represent $X \backslash S$ with $S=\{x, y, z\}$. (c): The black points represent $X \backslash S^{\prime}$ with $S^{\prime}=\{w\}$.

we replace the set $X$ by, for instance, the set $X \backslash S$ where $S=\{x, y, z\}$, we obtain a set which separates its complementary set into three components, see Fig. 4b: we can also say that we "merged two components of $\bar{X}$ through $S$ ". This operation may be seen as an "elementary merging" in the sense that only two components of $\bar{X}$ were merged. On the opposite, replacing the set $X$ by the set $X \backslash S^{\prime}$ where $S^{\prime}=\{w\}$, see Fig. 4k, would merge three components of $\bar{X}$. We also see that the component of $\bar{X}$ which is below $w$ (in light gray) cannot be merged by an "elementary merging" since any attempt to merge it must involve the point $w$, and thus also the three components of $\bar{X}$ adjacent to this point. In this section, we introduce definitions and basic properties related to such merging operations in graphs.

Definition 5. Let $(E, \Gamma)$ be a graph, $X \subset E$ and $S \subseteq X$. We say that $S$ is F-simple (for $X$ ) if $S$ is adjacent to exactly two components $A, B \in \mathcal{C}(\bar{X})$ such that $A \cup B \cup S$ is connected.

Let $p \in X$. We say that $p$ is F-simple (for $X$ ) if $\{p\}$ is F-simple for $X$.

In this definition, the prefix "F-" stands for fusion. For example, in Fig. 4 a, the point $z$ is F-simple while $x, y, w$ are not. Also, the sets $\{z\},\{x, y\},\{x, z\},\{y, z\}$, $\{x, y, z\}$ are $\mathrm{F}$-simple, but the sets $\{x\},\{y\}$ and $\{w\}$ are not.

Definition 6. Let $(E, \Gamma)$ be a graph and $X \subset E$. Let $A$ and $B \in \mathcal{C}(\bar{X})$, with $A \neq B$. We say that $A$ and $B$ can be merged (for $X$ ) if there exists $S \subseteq X$ such that $S$ is $F$-simple for $X$, and $A$ and $B$ are precisely the two components of $\bar{X}$ 
adjacent to $S$. In this case, we also say that $A$ and $B$ can be merged through $S$ (for $X$ ).

We say that $A$ can be merged (for $X$ ) if there exists $B \in \mathcal{C}(\bar{X})$ such that $A$ and $B$ can be merged for $X$.

For example, in Fig. 4h, the component of $\bar{X}$ in light gray cannot be merged, but each of the three white components can be merged for $X$.

\section{Fusion Graphs}

The preceding section and the present one constitute a theoretical basis for the study of region merging methods. The problems pointed out in the introduction can be avoided by using exclusively the notion of merging introduced in the previous section. In the sequel, we investigate several classes of graphs with respect to the possibility of "getting stuck" in a merging process.

Let $X \subset E$, and let $A, B \in \mathcal{C}(\bar{X})$. We set $\Gamma^{*}(A, B)=\Gamma^{*}(A) \cap \Gamma^{*}(B)$. We say that $A$ and $B$ are neighbors if $A \neq B$ and $\Gamma^{*}(A, B) \neq \emptyset$.

We begin with the definition of four classes of graphs.

Definition 7. We say that a graph $(E, \Gamma)$ is a weak fusion graph if, for any $X \subset E$ such that $|\mathcal{C}(\bar{X})| \geq 2$, there exist $A, B \in \mathcal{C}(\bar{X})$ which can be merged.

We say that a graph $(E, \Gamma)$ is a fusion graph if, for any $X \subset E$ such that $|\mathcal{C}(\bar{X})| \geq 2$, each $A \in \mathcal{C}(\bar{X})$ can be merged for $X$.

We say that the graph $(E, \Gamma)$ is a strong fusion graph if, for any $X \subset E$, any $A$ and $B \in \mathcal{C}(\bar{X})$ which are neighbors can be merged.

We say that the graph $(E, \Gamma)$ is a perfect fusion graph if, for any $X \subset E$, any $A$ and $B \in \mathcal{C}(\bar{X})$ which are neighbors can be merged through $\Gamma^{*}(A, B)$.

Basic examples and counter-examples of weak fusion, fusion, strong fusion, perfect fusion graphs and line graphs are given in Fig. 5 .

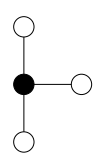

$(g)$

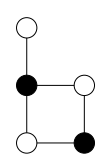

$(w)$

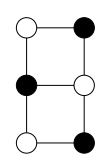

(f)

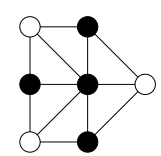

$(s)$

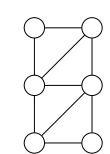

$(p)$

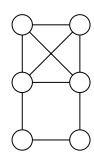

(l)

Fig. 5. Examples and counter-examples for different classes of graphs. $(g)$ : A graph which is not a weak fusion graph, $(w)$ : a weak fusion graph which is not a fusion graph, $(f)$ : a fusion graph which is not a strong fusion graph, $(s)$ : a strong fusion graph which is not a perfect fusion graph, $(p)$ : a perfect fusion graph which is not a line graph, and $(l)$ : a line graph. In the graphs $(g, w, f, s)$, the black vertices constitute a set $X$ which serves to prove that the graph does not belong to the pre-cited class.

We denote by $\mathcal{G}$ (resp. $\mathcal{G}_{L}, \mathcal{G}_{P}, \mathcal{G}_{S}, \mathcal{G}_{F}$, and $\mathcal{G}_{W}$ ) the set of all graphs (resp. line graphs, perfect fusion graphs, strong fusion graphs, fusion graphs, and weak fusion graphs). 
Property 2. We have the following strict inclusion relations:

$\mathcal{G} \supset \mathcal{G}_{W} \supset \mathcal{G}_{F} \supset \mathcal{G}_{S} \supset \mathcal{G}_{P} \supset \mathcal{G}_{L}$.

Now, we present the main theorem of this section, which establishes that the class of graphs for which any watershed is thin is precisely the class of fusion graphs. As an immediate consequence of this theorem and Prop. 2, we see that all watersheds in strong fusion graphs, perfect fusion graphs and line graphs are also thin.

Theorem 3. A graph $G$ is a fusion graph if and only if any non-trivial watershed in $G$ is thin.

Observe that the graphs of Figs. 3r,d,e are not fusion graphs; we see that they may indeed contain a non-thin watershed.

We conclude this section with a nice property of perfect fusion graphs, which can be useful to design hierarchical segmentation methods based on regions splitting. Consider the example of Fig. 6a, where a watershed $X$ (black points) in the graph $G$ separates $\bar{X}$ into two components. Consider now the set $Y$ (gray points) which is a watershed in the subgraph of $G$ induced by one of these components. We can see that the union of the watersheds, $X \cup Y$, is not a watershed, since the point $x$ is $\mathrm{W}$-simple for $X \cup Y$. Prop. 4 shows that this problem cannot occur in any perfect fusion graph.

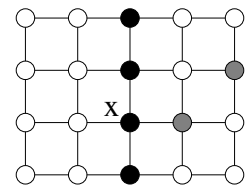

(a)

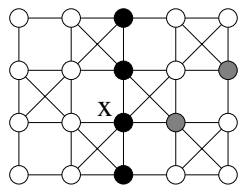

(b)

Fig. 6. Illustrations for Prop. 4 (a): The graph is not a perfect fusion graph (see Sec. 5. Prop. 8), and the union of the watersheds is not a watershed. (b): The graph is a perfect fusion graph (see Sec. 6. Prop. 10), the property holds.

Property 4. Let $G=(E, \Gamma)$ be a graph. If $G$ is a perfect fusion graph, then for any watershed $X \subset E$ in $G$ and for any watershed $Y \subset A$ in $G_{A}$, where $A \in \mathcal{C}(\bar{X})$ and $G_{A}$ is the subgraph of $G$ induced by $A$, the set $X \cup Y$ is a watershed in $G$.

\section{Local Characterizations}

Weak fusion, fusion, strong fusion and perfect fusion graphs are defined by conditions that must be verified for all the subsets of the vertex set. Thus, using the straightforward method based on the definition to check whether a graph belongs to one of these classes costs an exponential time with respect to the number of vertices. On the other hand, line graphs may be recognized thanks to a condition which can be checked independently in a limited neighborhood 
(informally speaking) of each vertex. Do such characterizations exist for the four classes of fusion graphs?

Let $(E, \Gamma)$ be a graph, let $x \in E$ and $k \in \mathbb{N}$, we denote by $\Gamma^{k}(x)$ the $k^{\text {th }}$ order neighborhood of $x$, that is, $\Gamma^{k}(x)=\Gamma\left(\Gamma^{k-1}(x)\right)$, with $\Gamma^{0}(x)=\{x\}$. We say that there is a local characterization of a class of graphs if there exists $k$ an arbitrary positive integer and $\mathcal{P}$ a property on graphs such that a graph $G=(E, \Gamma)$ is in this class if and only if for all $x \in E, \mathcal{P}[G(x, k)]$ is true, $G(x, k)$ being the subgraph of $G$ induced by $\Gamma^{k}(x)$.

Property 5. i) There is no local characterization of weak fusion graphs.

ii) There is no local characterization of fusion graphs.

Let $x$ and $y$ be two points, we say that $x$ and $y$ are 2-adjacent if $y \notin \Gamma(x)$ and $\Gamma^{*}(x) \cap \Gamma^{*}(y) \neq \emptyset$.

Theorem 6. Let $G=(E, \Gamma)$ be a graph. The graph $G$ is a strong fusion graph if and only if, for any two points $x, y \in E$ which are 2-adjacent, there exists $a \in \Gamma^{*}(x)$ and $b \in \Gamma^{*}(y)$ such that $b \in \Gamma(a)$ and $\Gamma(\{a, b\}) \subseteq[\Gamma(x) \cup \Gamma(y)]$.

Remind that in perfect fusion graphs, any two components $A, B$ of $\mathcal{C}(\bar{X})$ which are neighbors can be merged through $\Gamma^{*}(A) \cap \Gamma^{*}(B)$. Thus, perfect fusion graphs constitute an ideal framework for region merging methods. In the sequel, we will use the symbol $G^{\mathbf{\Delta}}$ to denote the graph (i) in Fig. 20b.

Theorem 7. Let $(E, \Gamma)$ be a graph.

The eight following statements are equivalent:

i) $(E, \Gamma)$ is a perfect fusion graph;

ii) for any $x \in E$, any $X \subseteq \Gamma(x)$ contains at most two connected components;

iii) for any non-trivial watershed $Y$ in $E$, each point $x$ in $Y$ is F-simple;

iv) for any connected subset $A$ of $E$, the subgraph of $(E, \Gamma)$ induced by $A$ is a fusion graph;

v) for any subset $X$ of $E$, there is no multiple point for $X$;

vi) the graph $G^{\mathbf{\Delta}}$ is not a subgraph of $G$;

vii) any vertices $x, y, z$ which are mutually non-adjacent are such that $\Gamma(x) \cap$ $\Gamma(y) \cap \Gamma(z)=\emptyset$;

viii) for any $x, y \in E$ which are 2-adjacent, for any $z \in \Gamma^{*}(x) \cap \Gamma^{*}(y)$, we have $\Gamma(z) \subseteq[\Gamma(x) \cup \Gamma(y)]$.

Notice that statement viii bears a resemblance with the local characterization of strong fusion graphs (Th. 6). Remind that any line graph is a perfect fusion graph (Prop. 2). We can see that, thanks to Th. 7 (condition vi), perfect fusion graphs can be characterized in a way similar to Th. 1 which characterizes line graphs, but with a much simpler condition. Remark, for example, that all the graphs of Fig. $2 \mathrm{~b}$ except $G^{\mathbf{\Delta}}$ are perfect fusion graphs, since none of these graphs contains $G^{\mathbf{\Delta}}$ as a subgraph.

\section{$5 \quad$ Usual Grids}

The aim of this section is to classify (with respect to the classes of fusion graphs) the grids which are the most commonly used in image processing. We prove 
that none of the grids commonly used in 2-dimensional and 3-dimensional image processing is a perfect fusion graph; several are not even fusion graphs. Thus, the most natural merging operation, which consists in merging two regions through their common boundary, is not a safe operation in these grids.

In this section, we will assume that $n$ is a strictly positive integer.

Let $E$ be a set and let $E^{n}$ be the Cartesian product of $n$ copies of $E$. An element $x$ of $E^{n}$ may be seen as a map from $\{1, \ldots, n\}$ to $E$, for each $i \in\{1, \ldots, n\}$, $x_{i}$ is the $i$-th coordinate of $x$.

Let $\mathbb{Z}$ be the set of integers. We consider the families of sets $H_{0}^{1}, H_{1}^{1}$ such that $H_{0}^{1}=\{\{a\} \mid a \in \mathbb{Z}\}, H_{1}^{1}=\{\{a, a+1\} \mid a \in \mathbb{Z}\}$. A subset $S$ of $\mathbb{Z}^{n}$ which is the Cartesian product of exactly $m \leq n$ elements of $H_{1}^{1}$ and $(n-m)$ elements of $H_{0}^{1}$ is called an $m$-cube.

In order to recover a graph structure for digital images, adjacency relations are defined on $\mathbb{Z}^{n}$. The following definition allows to retrieve the most frequently used adjacency relations.

Let $m \leq n$, we say that $x$ and $y$ in $\mathbb{Z}^{n}$ are $m$-adjacent if there exists an $m$-cube that contains both $x$ and $y$. We define $\Gamma_{m}^{n}$ as the binary relation on $\mathbb{Z}^{n}$ such that for any pair $x, y$ in $\mathbb{Z}^{n},(x, y) \in \Gamma_{m}^{n}$ if and only if $x$ and $y$ are $m$-adjacent.

In order to deal with graphs that can be arbitrarily large we define a grid as a pair $(E, \Gamma)$ where $E$ is an infinite set and $\Gamma$ is a binary relation on $E$. Let $X \subseteq E$ we define the restriction of $(E, \Gamma)$ to $X$ as the pair $\left(X, \Gamma_{X}\right)$ where $\Gamma_{X}=\Gamma \cap(X \times X)$. If $X$ is a finite set then $\left(X, \Gamma_{X}\right)$ is a graph. In the sequel, to simplify the notations, we will write $\Gamma$ as a shortcut for $\Gamma_{X}$. We first examine the case of 2-dimensional usual grids.

Property 8. Let $w, h$ be two integers such that $w>2$ and $h>2$. Let $E=\{x \in$ $\mathbb{Z}^{2} \mid 0 \leq x_{1}<w$ and $\left.0 \leq x_{2}<h\right\}$.

i) If $\{w, h\} \neq\{3,4\},\left(\bar{E}, \Gamma_{1}^{2}\right)$ is not a weak fusion graph. If $\{w, h\}=\{3,4\}$ then $\left(E, \Gamma_{1}^{2}\right)$ is a weak fusion graph but not a fusion graph.

ii) The graph $\left(E, \Gamma_{2}^{2}\right)$ is a fusion graph but is not a strong fusion graph.

Notice that in the literature, the graph $\left(E, \Gamma_{1}^{2}\right)$ (resp. $\left.\left(E, \Gamma_{2}^{2}\right)\right)$ corresponds to the 4 (resp. 8)-adjacency.

Now we examine the case of 3 -dimensional usual grids.

Property 9. Let $w, h$ and $d$ be three integers strictly greater than 1 . Let $E=$ $\left\{x \in \mathbb{Z}^{3} \mid 0 \leq x_{1}<w, 0 \leq x_{2}<h\right.$ and $\left.0 \leq x_{3}<d\right\}$.

i) The graph $\left(E, \Gamma_{1}^{3}\right)$ is not a weak fusion graph.

ii) If $w \geq 5, h \geq 5, d \geq 5$, the graph $\left(E, \Gamma_{3}^{3}\right)$ is not a fusion graph.

Notice that in the literature, the graph $\left(E, \Gamma_{1}^{3}\right)$ (resp. $\left.\left(E, \Gamma_{3}^{3}\right)\right)$ corresponds to the 6 (resp. 26)-adjacency.

\section{$6 \quad$ Perfect Fusion Grids}

We now introduce a grid for structuring $n$-dimensional digital images and prove that it is a perfect fusion graph, whatever the dimension $n$. 


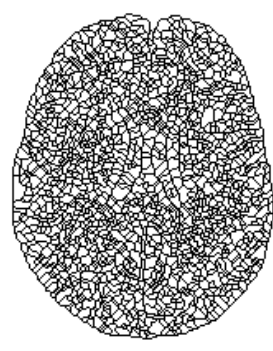

(a)

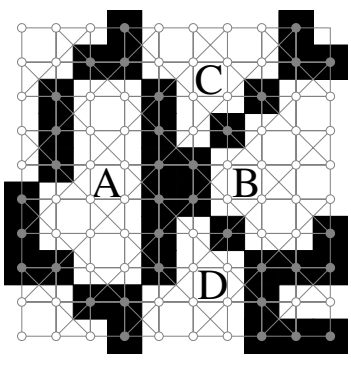

(b)

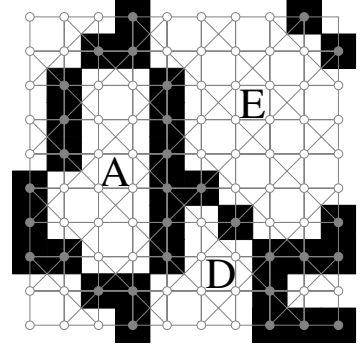

(c)

Fig. 7. (a): A watershed of Fig. 11 obtained on the perfect fusion grid; $(b)$ : a crop of (a) where the regions $A, B, C$ and $D$ correspond to the regions shown in Fig. 11; in gray, the corresponding perfect fusion grid is superimposed; $(c)$ : same as $(b)$ after having merged $B$ and $C$ to form a new region, called $E$.

It does thus constitute a structure on which neighboring regions can always be merged through their common neighborhood without problem with other regions. Fig. $8 \mathrm{~b}$ gives an intuitive idea of this grid. Fig. $7 \mathrm{a}$ shows a watershed of Fig. 1a obtained on this grid. Remark that the problems pointed out in the introduction do not exist in this case. The watershed does not contain any inner point. Any pair of neighboring regions can be merged by simply removing from the watershed the points which are adjacent to both regions (Figs. 7b,c). Furthermore, the resulting set is still a watershed. Observe that this grid is "between" the usual grids. In a forthcoming paper, we intend to prove that this is the unique such graph.

Let $C^{n}$ be the set of all n-cubes of $\mathbb{Z}^{n}$, we define the map $B$ from $C^{n}$ to $\mathbb{Z}^{n}$, such that for any $c \in C^{n}, B(c)_{i}=\min \left\{x_{i} \mid x \in c\right\}$, where $B(c)_{i}$ is the $i$-th coordinate of $B(c)$. It may be seen that $c$ is equal to the Cartesian product: $\left\{B(c)_{1}, B(c)_{1}+1\right\} \times \ldots \times\left\{B(c)_{n}, B(c)_{n}+1\right\}$. Thus clearly $B$ is a bijection.

We set $\mathbb{B}=\{0,1\}$. We set $\overline{0}=1$ and $\overline{1}=0$. A binary word of length $n$ is an element of $\mathbb{B}^{n}$. If $u$ is in $\mathbb{B}^{n}$, we define the complement of $u$ as the binary word $\bar{u}$ such that for any $i \in\{1, \ldots, n\},(\bar{u})_{i}=\left(\overline{u_{i}}\right)$.

Definition 8. Let $f$ be the map from $C^{n}$ to $\mathbb{B}^{n}$ such that for any $c \in C^{n}, f(c)_{i}$ is equal to $B(c)_{i} \bmod 2$, that is the remainder in the integer division of $B(c)_{i}$ by 2 .

Let $u$ be an element of $\mathbb{B}^{n}$, we set $C_{u}^{n}=\left\{c \in C^{n} \mid f(c)=u\right\}$ and $C_{u / \bar{u}}^{n}=$ $C_{u}^{n} \cup C_{\bar{u}}^{n}$.

We define the binary relation $\Gamma_{u / \bar{u}}^{n} \subseteq \mathbb{Z}^{n} \times \mathbb{Z}^{n}$ as the set of pairs $(x, y) \in$ $\mathbb{Z}^{n} \times \mathbb{Z}^{n}$ such that there exists $c \in C_{u / \bar{u}}^{n}$ that contains both $x$ and $y$.

We define $\mathcal{P}^{n}$, the family of perfect fusion grids over $\mathbb{Z}^{n}$, as the set $\mathcal{P}^{n}=$ $\left\{\left(\mathbb{Z}^{n}, \Gamma_{u / \bar{u}}^{n}\right) \mid u \in \mathbb{B}^{n}\right\}$.

Fig. 8 illustrates the above definitions for the two-dimensional case. Fig. 9 shows a watershed on a 3-dimensional perfect fusion grid. To clarify the figure, we use the following convention: any two points belonging to a same cube marked by a gray stripe are adjacent to each other. 


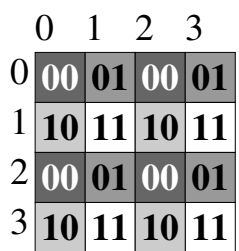

(a)

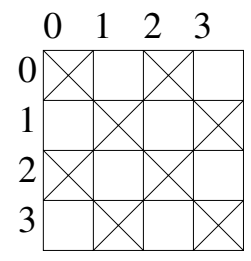

(b)

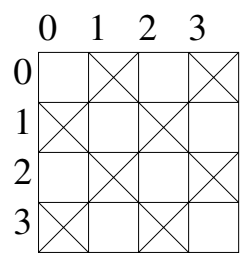

(c)

Fig. 8. Illustration of the two perfect fusions grids over $\mathbb{Z}^{2}$ (restricted to subsets of $\left.\mathbb{Z}^{2}\right)$. (a): The map $f ;(b):\left(\mathbb{Z}^{2}, \Gamma_{11 / 00}^{2}\right) ;(c):\left(\mathbb{Z}^{2}, \Gamma_{10 / 01}^{2}\right)$.
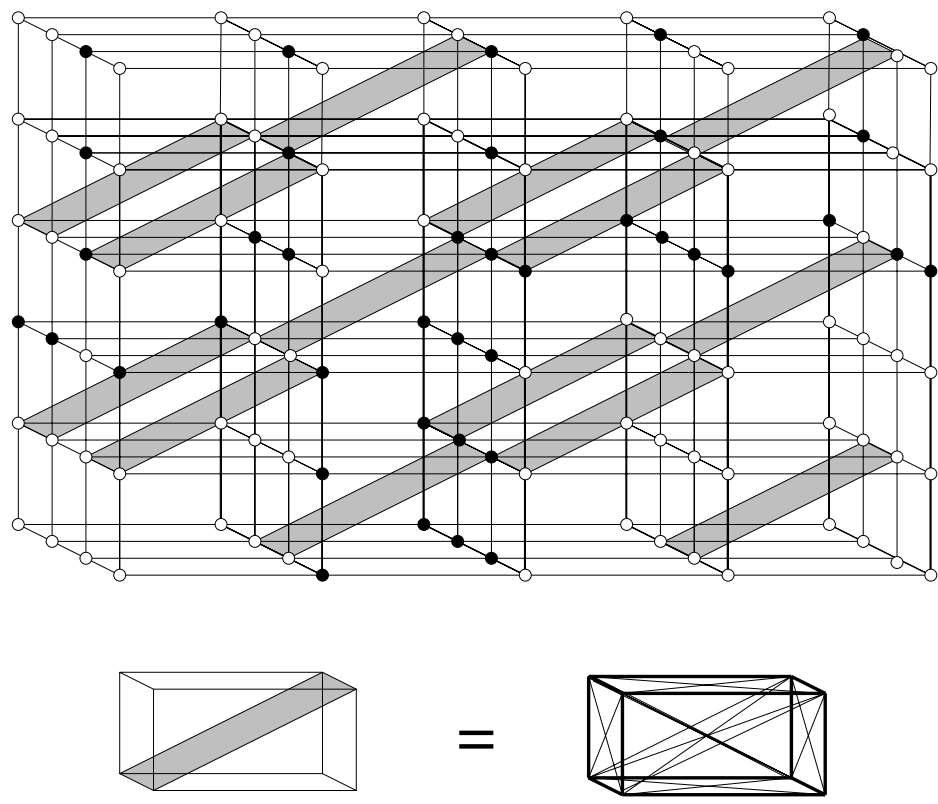

Fig. 9. A 3-dimensional perfect fusion grid. Black points constitute a set which is a watershed.

Theorem 10. Let $u \in \mathbb{B}^{n}$ and let $X$ be a finite subset of $\mathbb{Z}^{n}$ such that $\left(X, \Gamma_{u / \bar{u}}^{n}\right)$ is connected. Then $\left(X, \Gamma_{u / \bar{u}}^{n}\right)$ is a perfect fusion graph such that $\Gamma_{1}^{n} \subseteq \Gamma_{u / \bar{u}}^{n} \subseteq \Gamma_{n}^{n}$. Furthermore it is a line graph.

It may be seen that the family $\mathcal{P}^{n}$ contains $2^{n-1}$ distinct perfect fusion grids.

Let $X \subseteq \mathbb{Z}^{n}$ and let $t \in \mathbb{B}^{n}$. We define $X+t=\{x+t \mid x \in X\}$, we say that $X+t$ is a binary translation of $X$. Let $m$ be a positive integer such that $m \leq n$. Remark that if $X$ is an m-cube then $X+t$ is also an m-cube.

Let $u$ and $v$ in $\mathbb{B}^{n}$. Let $t \in \mathbb{B}^{n}$ such that for any $i \in\{1, \ldots, n\}$, if $u_{i}=\overline{v_{i}}$ then $t_{i}=1$, otherwise $t_{i}=0$. Then for any $(x, y) \in \mathbb{Z}^{n} \times \mathbb{Z}^{n},(x, y) \in \Gamma_{u / \bar{u}}^{n}$ if and only if $(x+t, y+t) \in \Gamma_{v / \bar{v}}^{n}$. 
In words, any two n-dimensional perfect fusion grids are equivalent up to a binary translation.

\section{Conclusion and Perspectives}

This article sets up a theoretical framework for the study of merging properties in graphs. Forthcoming articles ( [13] for example) extend this study to the case of weighted graphs, which constitute a model for grayscale images. The notion of topological watershed [7,6] extends the notion of watershed to weighted graphs, and possesses interesting properties which are not guaranteed by most watershed algorithms [9]. The major outcomes of [13] are:

i) a proof that any topological watershed on any perfect fusion graph is thin;

ii) a new, simple and linear-time algorithm to compute topological watersheds on perfect fusion graphs.

\section{References}

1. Rosenfeld, A., Kak, A.: 10. In: Digital picture processing. Volume 2. Academic Press (1982) Section 10.4.2.d (region merging).

2. Pavlidis, T.: 4-5. In: Structural Pattern Recognition. Volume 1 of Springer Series in Electrophysics. Springer-Verlag (1977) 90-123 (segmentation techniques).

3. Beucher, S., Lantuéjoul, C.: Use of watersheds in contour detection. In: procs. Int Workshop on Image Processing Real-Time Edge and Motion Detection/Estimation. (1979)

4. Vincent, L., Soille, P.: Watersheds in digital spaces: An efficient algorithm based on immersion simulations. PAMI 13(6) (1991) 583-598

5. Meyer, F.: Un algorithme optimal de ligne de partage des eaux. In: Actes du 8ème Congrès AFCET, Lyon-Villeurbanne, France (1991) 847-859

6. Couprie, M., Bertrand, G.: Topological grayscale watershed transform. In: SPIE Vision Geometry V Proceedings. Volume 3168. (1997) 136-146

7. Bertrand, G.: On topological watersheds. JMIV 22(2-3) (2005) 217-230

8. Couprie, M., Najman, L., Bertrand, G.: Quasi-linear algorithms for the topological watershed. JMIV 22(2-3) (2005) 231-249

9. Najman, L., Couprie, M., Bertrand, G.: Watersheds, mosaics and the emergence paradigm. DAM 147(2-3) (2005) 301-324

10. Jasiobedzki, P., Taylor, C., Brunt, J.: Automated analysis of retinal images. IVC 1(3) (1993) 139-144

11. Cousty, J., Bertrand, G., Couprie, M., Najman, L.: Fusion graphs: merging properties and watershed. CVIU (2006) submitted. Also in technical report IGM2005-04, http://igm.univ-mlv.fr/LabInfo/rapportsInternes/2005/04.pdf.

12. Beineke, L.: On derived graphs and digraphs. In Sachs, H., Voss, H., Walther, H., eds.: Beiträge zur graphen theorie. Teubner (1968) 17-23

13. Cousty, J., Couprie, M., Najman, L., Bertrand, G.: Grayscale watersheds on perfect fusion graphs. In: procs. IWCIA. (2006) 